\title{
Initial clinical results with a fusion prototype for mammography and three-dimensional ultrasound with a standard mammography system and a standard ultrasound probe
}

Acta Radiologica

2018, Vol. 59(12) 1406-1413

(C) The Foundation Acta Radiologica 2018

Article reuse guidelines:

sagepub.com/journals-permissions DOI: $10.1177 / 0284185118762249$ journals.sagepub.com/home/acr (SAGE

\author{
Julius Emons', Marius Wunderle', Arndt Hartmann', \\ Marcus Radicke ${ }^{3}$, Claudia Rauh', Michael Uder ${ }^{4}$, \\ Paul Gass' ${ }^{\infty}$, Peter A Fasching', Hanna Langemann', \\ Matthias W Beckmann', Rüdiger Schulz-Wendtland ${ }^{4}{ }^{4}$ \\ and Sebastian M Jud' ${ }^{1, *} \mathbb{C}$
}

\begin{abstract}
Background: Combinations of different imaging techniques in fusion devices appear to be associated with improvements in diagnostic assessment.

Purpose: The aim of this study was to test the feasibility of using an automated standard three-dimensional (3D) ultrasound (US) device fused with standard mammography for the first time in breast cancer patients.

Material and Methods: Digital mammograms and 3D automated US images were obtained in 23 patients with highly suspicious breast lesions. A recently developed fusion machine consisting of an ABVS 3D US transducer from an Acuson S2000 machine and a conventional Mammomat Inspiration device (both Siemens Healthcare GmbH, Erlangen, Germany) were used for the purpose. The feasibility of the examinations, imaging coverage, and patients' experience of the procedure were examined.

Results: In 15 out of 19 patients, the region of interest (ROI) with the tumor marked in the mammogram was visible on US. The examination was experienced positively by the patients, with no unexpected pain or injury. The examination was time-saving and well tolerated.

Conclusion: In conclusion, we have shown initial clinical feasibility of an US/radiography fusion prototype with good localization and evaluation of the ROls. The combined examination was well tolerated. The simultaneous evaluation with mammography and US imaging may be able to improve detection and reduce examiner-related variability.
\end{abstract}

\section{Keywords}

Mammography, automated breast ultrasound, multimodal breast imaging

Date received: 27 June 2017; accepted: 7 February 2018

\section{Introduction}

Although tremendous progress has been made in our knowledge of risk factors and genetic predispositions for breast cancer, the majority of breast cancer cases

'Department of Gynecology and Obstetrics, Erlangen University Hospital, Comprehensive Cancer Center Erlangen-EMN, Friedrich Alexander University of Erlangen-Nuremberg, Erlangen, Germany
${ }^{2}$ Institute of Pathology, Erlangen University Hospital, Comprehensive Cancer Center Erlangen-EMN, Friedrich Alexander University of Erlangen-Nuremberg, Erlangen, Germany

${ }^{3}$ Siemens Healthcare GmbH, Erlangen, Germany

${ }^{4}$ Institute of Diagnostic Radiology, Erlangen University Hospital, Erlangen, Germany

*Equal contributors.

Corresponding author:

Sebastian M Jud, Department of Gynecology and Obstetrics, Erlangen University Hospital, Comprehensive Cancer Center Erlangen-EMN, Universitätsstrasse 21-23, 91054 Erlangen, Germany. Email: sebastian.jud@uk-erlangen.de 
occur without any known predictive factors (1). Screening mammography and early detection are still the primary strategy for reducing the mortality rate from breast cancer (2).

Most patients with breast cancer present with a palpable tumor. Not all lesions are therefore identified on screening mammography and the sensitivity of mammography may be compromised in breasts that have higher mammographic density (3-7). In some countries, it is therefore obligatory for women who have dense breast tissue to have information about breast density included on their federally required mammography reports. As a consequence, ultrasonography may also be performed in addition to mammography (8). However, although not every woman with a high breast density needs to undergo an ultrasound (US) examination as well (9), there are other factors independent of mammographic density that can identify women who may benefit from an additional US examination (6).

Ultrasonography is performed routinely in addition to mammography. When mammography alone is compared with mammography plus US, the sensitivity and specificity change from $50 \%$ and $95 \%$ for mammography alone to $76 \%$ and $89 \%$ for the combination of the two imaging modalities $(2-5,10)$.

A major requirement for multimodal breast imaging is accurate localization of the specific lesions with the different modalities and this can be challenging. Mammography is performed in an upright position with compressed breast tissue and it provides twodimensional (2D) imaging. US offers better, more three-dimensional (3D) localization and is strongly dependent on each US examiner's individual level of experience $(5,11,12)$. In addition, conventional mammography and US are performed one after another and this may lead to difficulties particularly with the correct localization of different lesions, (13). Studies investigating the consistency of regions of interest (ROIs) in mammography and freehand ultrasonography have reported that incorrect localization occurs in around $10 \%$ of cases (14). As a consequence of these difficulties, various imaging devices for performing mammography and US simultaneously have been investigated and developed (15-19).

This paper describes the development and use of a combined imaging system featuring digital mammography with an attached automated US scanner providing 3D imaging.

\section{Material and Methods}

\section{Patients}

The patients provided written informed consent and the study was approved by the ethics committee of the
Medical Faculty of the University of ErlangenNuremberg. Patients were recruited from the diagnostic breast cancer unit at the University Breast Center Franconia in the Comprehensive Cancer Center Erlangen-EMN. Patients were eligible for inclusion in the study if they had been referred to the breast cancer unit for further assessment with a lesion that was highly suspicious on clinical diagnosis or imaging. The patients also had to be aged at least 18 years. No other inclusion or exclusion criteria were applied.

\section{US/X-ray fusion prototype}

All patients were examined with an US/mammography prototype, developed from a standard mammography machine and a standard 3D US probe, as previously described (20). The machine is illustrated in Suppl. Fig. 1. Briefly, the upper compression plate was replaced by a frame into which an elastic gauze was mounted. The gauze was able to provide compression up to 100 Newton. This upper compression plate was flangemounted onto a Mammomat Inspiration machine (Siemens Healthcare GmbH, Erlangen, Germany) instead of a standard compression plate (20). The US probe from an Acuson S2000 automated breast volume scanner (ABVS; Siemens Healthcare $\mathrm{GmbH}$,) was mounted into the frame and was able to capture 3D US images through the gauze from above the breast (20). The prototype underwent normal safety tests, which were based on the relevant subclauses of the International Electrotechnical Commission's safety standard IEC 60601. Additionally, compression of the breast was previously tested in a set of five mastectomy specimens (20) to investigate the optimal compression force. The breast was compressed to the required thickness for a standard mammography image at up to $100 \mathrm{~N}$ by the taut elastic gauze fixed to the underside of the compression frame. The time required for acquisition of the mammography and automated US data averaged $70 \mathrm{~s}$, as previously described (20).

The prototype used in this study is a research device and is not commercially available.

\section{Imaging procedure}

All patients underwent positioning and fixation of the breast for a craniocaudal image. The breast was compressed using the taut gauze instead of a stiff compression plate. This gave the breast a slightly convex shape, reduced X-ray absorption by the compression device (with gauze instead of a plate), and provided better US coupling in comparison with a standard compression plate. The US volume was obtained by automatically moving the transducer from lateral to medial. During $\mathrm{X}$-ray imaging, the US transducer remained outside 
the X-ray field. The breast was compressed to the required thickness for a standard mammography image, based on the individual breast shape. Craniocaudal mammography was performed with a standard protocol derived from routine clinical work, followed by an automated 3D US scan. Between the X-ray and US imaging procedures, a coupling lotion was spread on top of the gauze.

The images were manually copied to a separate computer and a fixed-image correlation was inserted into the DICOM header. Reading was performed with a prototype software program developed by MEVIS Breastcare GmbH, Bremen, Germany.

\section{Assessment of images}

The image sets containing the craniocaudal mammogram, the US cross-section, and the reconstructed 3D US images were presented to two independent readers with more than ten years of experience of using the software described above. First, the density of the breast was categorized on a scale of 1-4 in accordance with the American College of Radiology classification, and the longitudinal nipple-pectoralis distance was measured as illustrated in Suppl. Fig. 2. The suspicious lesion was then identified on the mammogram, and its length and width were measured. The software features cross hairs for simultaneous navigation in all three image types (B-mode US, reconstructed $3 \mathrm{D}$ imaging, and mammography). The cross hairs were placed in the middle of the lesion in the mammogram and the corresponding finding was measured on the 3D US image.

The sagittal coverage of the breast on the mammogram images and 3D US was measured as follows. The reader marked the shortest distance between the nipple and the pectoralis muscle or image cut-off line on both the mammogram and the 3D US image. The distance was measured, and the difference was used as a surrogate for estimating the area of the breast not covered by $3 \mathrm{D}$ US. The measurement method is illustrated in Suppl. Fig. 2 together with the full dataset. The quality of the mammogram images was not assessed in this study, as the results were similar to those obtained with standard mammography in a previous study (20). The quality of the US images was assessed independently by two experienced radiologists. As these images were only used for scientific purposes, the quality check focused on evaluating the visibility of the suspicious area due to insufficient coverage or artifacts.

\section{Clinical data}

The patient data were obtained prospectively. After informed consent had been received, the data were documented from patient charts and entered into an electronic case report form.

\section{Patient questionnaire and safety assessment}

The patients were asked to complete a questionnaire inquiring into any pain experienced and the comfort of the procedure, as well as any redness of the breast noticed after the examination. The detailed topics assessed are listed in Suppl. Table 2. The patients were also asked to indicate their overall experience of pain using a visual analog scale of $0-5$. Medical staff inspected the breast after the imaging procedure to document any occurrence of hematoma, bleeding, or other signs of injury to the breast. It was also documented whether the gauze was intact after the imaging procedure.

\section{Statistical analysis}

The data are presented descriptively. Patient and tumor data, questionnaire results, and examination characteristics are presented as totals and frequencies, means with standard deviation, or medians and ranges.

\section{Results}

\section{Patient and tumor characteristics}

A total of 23 women with 24 highly suspicious lesions were recruited for this study between March and May 2016. Five women were excluded because the US images were not stored, due to human error. The study population thus consisted of 18 women with 19 lesions. Fig. 1 shows the study flow chart.

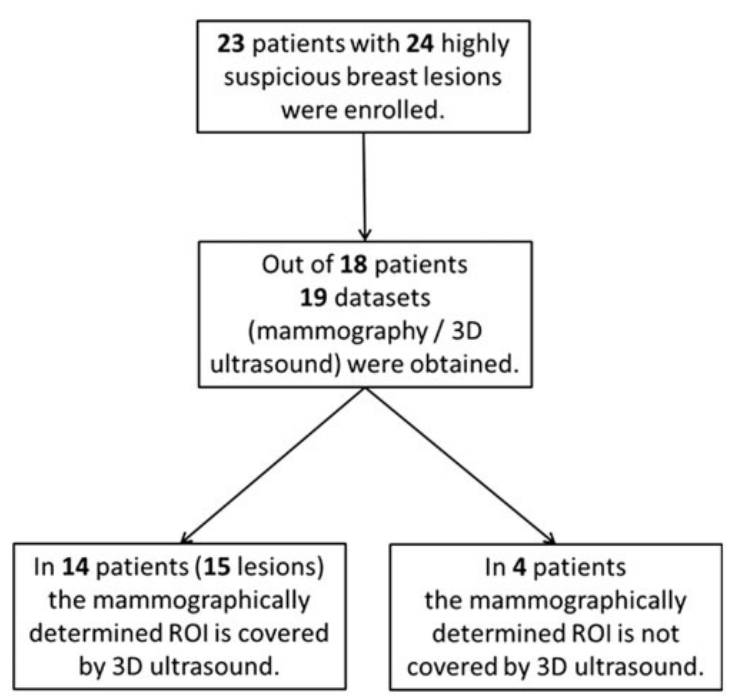

Fig. I. Numbers of patients and datasets. 
Table I. Characteristics of the patients and tumors.

\begin{tabular}{|c|c|c|}
\hline & $\mathrm{n}$ or mean & $\%$ or SD \\
\hline Patients (n) & 23 & \\
\hline Age at diagnosis (years) & 65.5 & 12.6 \\
\hline \multicolumn{3}{|l|}{ Age (years) } \\
\hline$<45$ & 1 & 4.3 \\
\hline $45-54$ & 3 & 13.0 \\
\hline$>54$ & 19 & 82.6 \\
\hline \multicolumn{3}{|l|}{ Histological type } \\
\hline No specific type & 23 & \\
\hline \multicolumn{3}{|l|}{ Tumor size $(\mathrm{cm})$} \\
\hline$\leq 2$ & 13 & 56.5 \\
\hline$>2$ & 10 & 43.4 \\
\hline \multicolumn{3}{|l|}{ Histological grade } \\
\hline I or 2 & 15 & 65.2 \\
\hline 3 & 8 & 34.7 \\
\hline \multicolumn{3}{|l|}{ Estrogen receptor status } \\
\hline Positive & 21 & 91.3 \\
\hline Negative & 2 & 8.7 \\
\hline \multicolumn{3}{|c|}{ Menopausal status at diagnosis } \\
\hline Premenopausal & 0 & \\
\hline Postmenopausal & 23 & 100.0 \\
\hline \multicolumn{3}{|l|}{ Nodal status } \\
\hline Positive & 4 & 17.3 \\
\hline Negative & 19 & 82.6 \\
\hline \multicolumn{3}{|l|}{ Body mass index $\left(\mathrm{kg} / \mathrm{m}^{2}\right)$} \\
\hline$\leq 25$ & II & 47.8 \\
\hline$>25$ & 12 & 52.1 \\
\hline
\end{tabular}

SD: standard deviation.

The women's mean age was 65.5 years (age range $=$ $39-91) ; 47.8 \%$ had a body mass index (BMI) $\leq 25$ and $52.1 \%$ had a $\mathrm{BMI}>25$. The patients were diagnosed and treated at the breast center in accordance with standard clinical practice. Invasive breast cancer was confirmed in all the patients investigated. Patient and tumor characteristics are listed in Table 1. Mammographic densities and tumor sizes are shown with the means for the two readers' assessments in Table 2.

\section{Image coverage}

In general, the coverage of the whole breast area on mammography was comparable to that with mammography alone. The US coverage was smaller. The prototype lacks $1.2 \mathrm{~cm}$ of US image coverage proximal to the chest wall, for constructional reasons. Measuring the coverage from the nipple to the pectoralis muscle empirically showed a consistently smaller distance on
Table 2. Overview of breast density, sagittal coverage, measured tumor size, and US coverage.

\begin{tabular}{lcc}
\hline & Mean or $\mathrm{n}$ & SD or \% \\
\hline Breast density (n/\%) & 5 & 21.7 \\
$\mathrm{I}$ & 8 & 34.7 \\
2 & 6 & 26.1 \\
3 & 0 & \\
4 & & \\
Sagittal coverage (mean in cm/SD) & 11.7 & 3.4 \\
Mammography & 7.6 & 2.6 \\
3D US & & 64.8 \\
Percentage coverage & & \\
Measured tumor size (mean in cm/SD) & & \\
Mammography & 1.6 & 1.2 \\
Length & 1.3 & 0.9 \\
Width & & \\
3D US & 1.0 & 0.7 \\
Length & 1.0 & 0.7 \\
Width &
\end{tabular}

SD: standard deviation; US: ultrasound.

US from the most anterior to the most posterior part of the breast, which was still captured on the other imaging modality (Fig. 4). The mean difference in the sagittal coverage between the two imaging modalities was $4.1 \mathrm{~cm}( \pm 1.6 \mathrm{~cm})$.

Regarding coverage and artifacts, the image quality in the ultrasonography was sufficient for detecting and describing all suspicious lesions and was therefore sufficient for this study - although, as seen in Figs. 2 and 3 , artifacts were still visible for computational reasons on the $3 \mathrm{D}$ images with the prototype's constructional set-up. The nipple area was not sufficiently covered with the technique.

\section{Feasibility}

Among the enrolled patients for whom full datasets including both image modalities were available, the suspicious lesions were visible on all mammograms. Using the software program, the US images were carefully examined in the regions in which the tumor was thought to be located. The tumors were clearly identifiable and localizable on the 3D US images in 15 cases. Two examples of cases with good visibility are shown in Fig. 2. In four cases (Fig. 3), the corresponding area was not captured on 3D US. In two of the four cases, the tumor was located subcutaneously in the nipple region. In the other two cases, the mammographic findings were located close to the pectoralis muscle. 

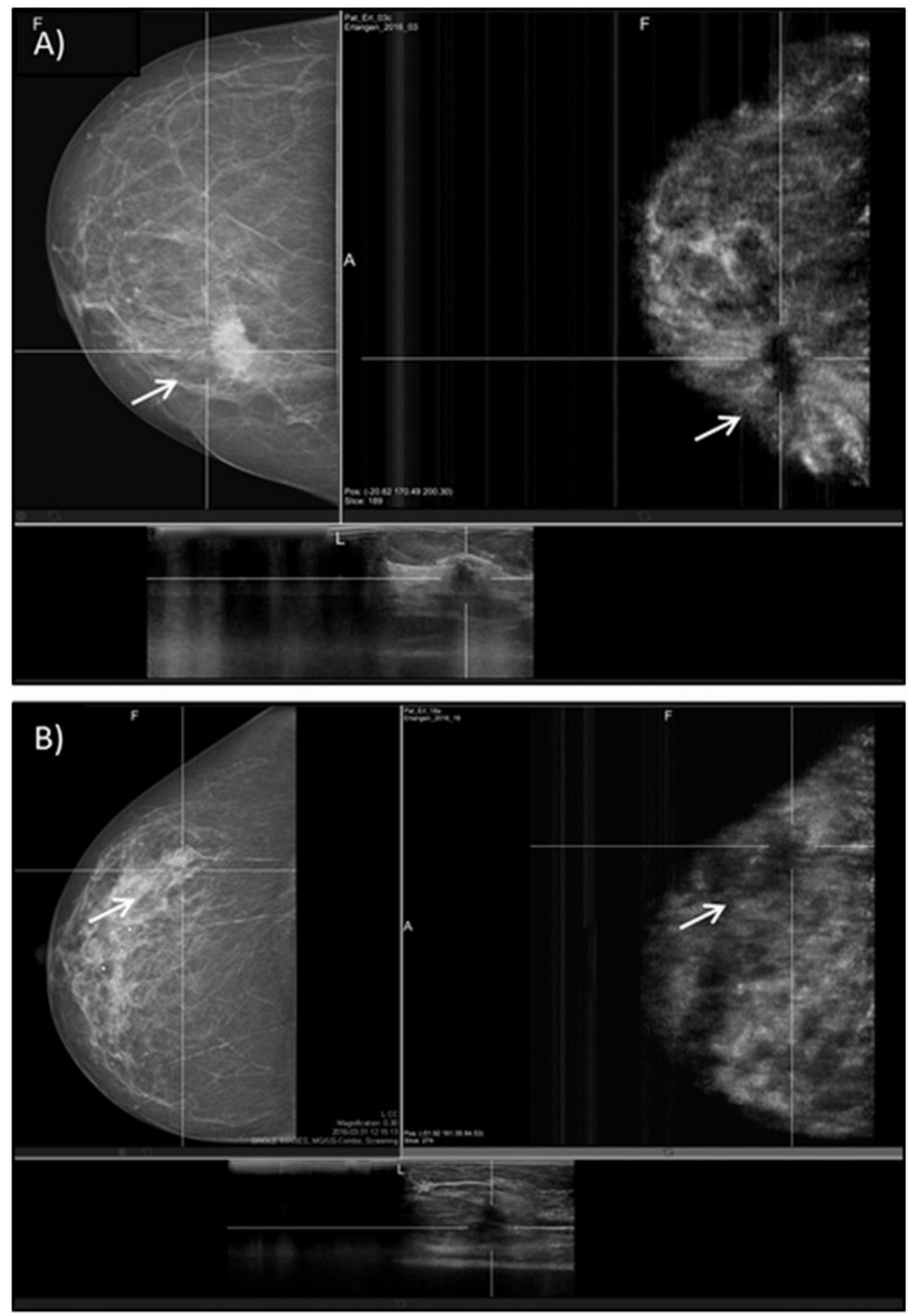

Fig. 2. Example image sets with combined ultrasonography and mammography. Left: mammography images. The ROI is marked with a white arrow. Right: the corresponding 3D-reconstructed breast, based on the cross-sectional 2D US images. The corresponding ROI is marked with a white arrow. Bottom: the cross-sectional 2D US image that is acquired almost simultaneously with the mammography. (a) Patient 3. Mammogram of a cT2 invasive carcinoma (NST) measuring $27 \times 21 \mathrm{~mm}$. Both the 2D image and the 3D US image clearly reproduce the finding, measuring $20 \times 12 \mathrm{~mm}$. (b) Patient 18. Mammogram of a cTI invasive carcinoma (NST) measuring $18 \times 13 \mathrm{~mm}$, with associated microcalcifications. The findings, measuring $15 \times 14 \mathrm{~mm}$, are clearly reproduced on the US images.

\section{Pain}

All 23 patients responded to the pain questionnaire. Thirty-nine percent of the patients reported that they felt pain during the investigation, with a mean pain score of 1.4 on a scale of 0 to 5 . Sixty-one percent of the patients report that they did not feel any pain, with a mean pain score of 0.8 . None of the patients suffered severe pain or injury. An overview of the survey of pain perception is given in Suppl. Table 1.

\section{Safety assessment}

Medical staff did not report any hematoma, bleeding, or other signs of injury after the investigation. The gauze was intact after all imaging procedure. 

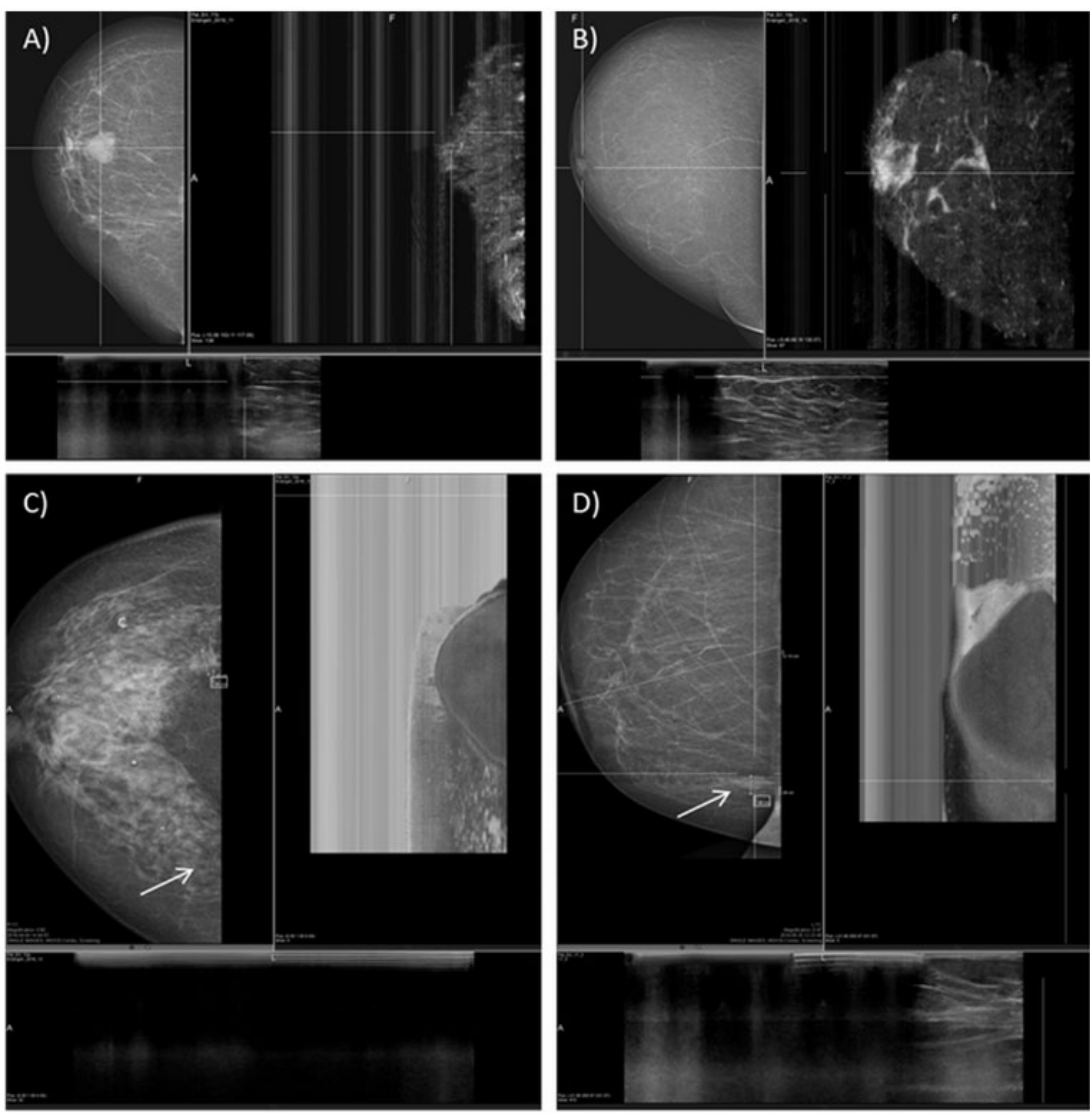

Fig. 3. Image sets for four patients with inadequate US coverage. (a, b) Patients II and I4. The mammographically detectable breast lesions in the nipple region (a) and subcutaneously (b) are marked with white arrows. In these examples, the 2D and 3D US imaging is not adequately covering the harder-to-compress nipple region. (c, d) Patients 12 and I7. Mammographic findings located close to the pectoralis muscle, which are not covered on the 2D and 3D US images. For technical reasons, the 3D US probe lacks approximately $1.2 \mathrm{~cm}$ of image coverage toward the chest.

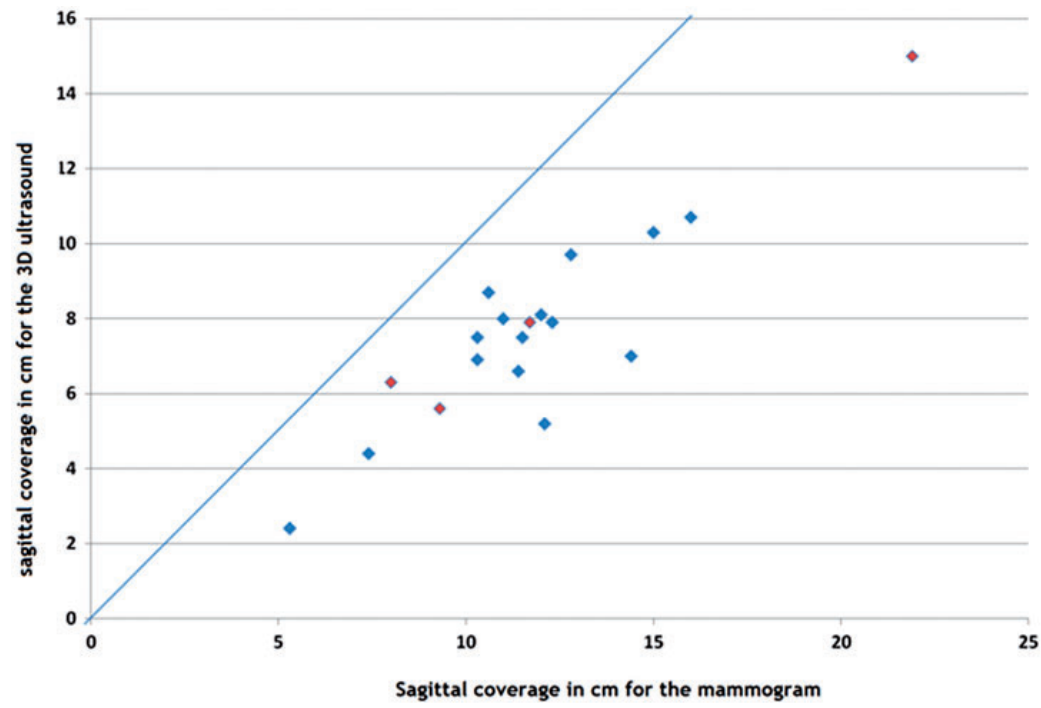

Fig. 4. Comparison of distances between the nipple and pectoralis measured with US and mammography. The graph shows the nipple-pectoralis distance with the two imaging modalities. Red dots represent cases in which the ROI was not covered by US. 


\section{Discussion}

This study examined the feasibility, US coverage, and imaging quality of a fusion prototype constructed from a digital mammography unit and a standard 3D US probe. The prototype allowed examinations of the breast to be carried out with mammography and 3D ultrasonography with a single fixation procedure for both examinations, making it possible to identify ROIs seen in each of the imaging modalities in the corresponding image from the other modality, using a software program. The imaging coverage provided by $3 \mathrm{D}$ US still needs to be optimized.

The method makes it possible to examine the compressed and fixed breast with two imaging techniques in a single procedure without changing the position or shape of the breast. The main advantages of the technique are the absence of positional changes; more precise detection of lesions, particularly in dense breast tissue; and reduced investigator-dependent variability and effort (21). In addition, simultaneous investigation reduces the impact of biological factors such as cyclical fibrocystic changes on the imaging quality, as previously reported by Kapur et al. (17).

Achieving complete coverage of the whole breast in both imaging modalities is a technical challenge. For signal transduction, US requires a certain amount of contact pressure on the tissue, which is complicated by the naturally convex shape of the breast. Conventional hand-held US is associated with good area coverage, resulting from the movement of the US probe in a skilled investigator's hand. This was not possible in the fusion prototype used in this study, due to the automated rigid application of the US probe. In addition, during conventional examination the investigator is able to adjust the pressure of the US probe to the natural individual shape of the breast, allowing continuous pressure to be applied and resulting in better imaging quality. In conventional ultrasonography, it is also possible to re-evaluate suspicious areas with varying pressure and from different angles, again improving the quality of the assessment. Both of these features are difficult to achieve with an automated approach. In the present study, subcutaneous coverage was thus impaired, particularly in the nipple region. The area close to the chest wall was also insufficiently covered. Inhomogeneous pressure also reduced the US transduction and increased imaging artifacts. On the other hand, automated US allows 3D reconstruction of the breast, is superior for investigator-independent re-localization of ROIs, and allows delayed interpretation at an observer-friendly monitor-based reading station (2).

In mammography, it is mandatory to apply adequate pressure to the breast in order to achieve satisfactory imaging quality (22). The optimal pressure application was previously assessed in a case series using a small number of mastectomy specimens (20). The prerequisite for adequate compression in the fusion prototype is homogeneous distribution of pressure, an absence of relevant distraction or absorption of radiation, and a high level of US transmittance. This goal was achieved by using a taut elastic gauze as described above, resulting in good mammographic quality (20). In line with these findings, other studies have shown that similar forms of compression using a compression gauze did not reduce the quality of mammographic imaging (23). In some of these imaging systems, the breast is immobilized and compressed by, and scanned through, a plastic paddle with various thicknesses (23). The imaging quality in these systems using a thin rigid plate was mainly limited by defocusing effects, and in the system with a thick plate the US beam was attenuated $(22,23)$. In the present study, it was found that there is less coverage in the areas behind the nipple area and in the prepectoral region; otherwise, no loss of US image intensity was noted.

To ensure well-balanced and comfortable pressure on the breast, an anatomically shaped gauze has been developed that fits the breast better than conventional mammography compression plates. This is of special importance, as imaging with the fusion prototype is more time-consuming with the two simultaneous imaging techniques on the compressed breast than with conventional mammography alone. Assessment of the extent of patients' comfort with compression during conventional mammography and with the anatomically shaped gauze showed similar results for patients' pain perception, although the compression time with the fusion prototype was longer in comparison with standard mammography.

The time required to carry out the mammography and automated US was a mean of $70 \mathrm{~s}(20)$, so that it is more time-saving than performing the two imaging modalities one after the other. The potentially low cost and ease of use of a combined mammography and automated US system in comparison with magnetic resonance imaging might make this method a good alternative, especially for patients who are at increased risk for breast cancer (2).

The fusion prototype still has limitations. The nipple and subcutaneous region, as well as the prepectoral region, are not yet satisfactorily covered by US. Current research is therefore being carried out to optimize US coverage of the whole breast. The study has assessed the feasibility and has shown acceptable coverage of the system. The limitations of the method may be improved using a reconfigured prototype with better coverage. Furthermore, there were no women with ACR 4 breasts density in our population, which should benefit the most by the additional US. This should be considered in a new study design. 
In conclusion, we have shown initial clinical feasibility of an US/mammography fusion prototype with good localization and evaluation of the ROIs. Further, the combined examination was well tolerated by the patients. The simultaneous evaluation with mammography and automated US imaging may be able to improve detection and reduce examiner-related variability. The aim for further development is to incorporate $3 \mathrm{D}$ imaging into the mammographic modality and replace mammography with tomosynthesis.

\section{Declaration of Conflicting Interests}

The author(s) declared the following potential conflicts of interest with regard to the research, authorship, and/or publication of this article: Marcus Radicke is an employee of Siemens Healthcare. He was not involved in the analysis of the data or the interpretation of the results.

\section{Funding}

The author(s) received no financial support for the research, authorship, and/or publication of this article.

\section{ORCID iD}

Paul Gass (D) http://orcid.org/0000-0002-4239-5528

Sebastian M Jud (D) http://orcid.org/0000-0002-8894-2785

\section{References}

1. Colditz GA, Willett WC, Hunter DJ, et al. Family history, age, and risk of breast cancer. Prospective data from the Nurses' Health Study. JAMA 1993;270:338-343.

2. Kelly KM, Dean J, Comulada WS, et al. Breast cancer detection using automated whole breast ultrasound and mammography in radiographically dense breasts. Eur Radiol 2010;20:734-742.

3. Mandelson MT, Oestreicher N, Porter PL, et al. Breast density as a predictor of mammographic detection: comparison of interval- and screen-detected cancers. JNCI 2000;92:1081-1087.

4. Kolb TM, Lichy J, Newhouse JH. Comparison of the performance of screening mammography, physical examination, and breast US and evaluation of factors that influence them: an analysis of 27,825 patient evaluations. Radiology 2002;225:165-175.

5. Berg WA, Blume JD, Cormack JB, et al. Combined screening with ultrasound and mammography vs mammography alone in women at elevated risk of breast cancer. JAMA 2008;299:2151-2163.

6. Haberle L, Fasching PA, Brehm B, et al. Mammographic density is the main correlate of tumors detected on ultrasound but not on mammography. Int J Cancer 2016;139: 1967-1974.

7. Boyd NF, Guo H, Martin LJ, et al. Mammographic density and the risk and detection of breast cancer. NEJM 2007;356:227-236.
8. Weigert JM. The Connecticut experiment; the third installment: 4 years of screening women with dense breasts with bilateral ultrasound. Breast J 2017;23:34-39.

9. Burkett BJ, Hanemann CW. A review of supplemental screening ultrasound for breast cancer: certain populations of women with dense breast tissue may benefit. Acad Radiol 2016;23:1604-1609.

10. Schaefer FK, Waldmann A, Katalinic A, et al. Influence of additional breast ultrasound on cancer detection in a cohort study for quality assurance in breast diagnosisanalysis of 102,577 diagnostic procedures. Eur Radiol 2010;20:1085-1092.

11. Buchberger W, DeKoekkoek-Doll P, Springer P, et al. Incidental findings on sonography of the breast: clinical significance and diagnostic workup. Am J Roentgenol 1999;173:921-927.

12. Kopans DB. Breast-cancer screening with ultrasonography. Lancet 1999;354:2096-2097.

13. Skaane P, Engedal K, Skjennald A. Interobserver variation in the interpretation of breast imaging: comparison of mammography, ultrasonography, and both combined in the interpretation of palpable noncalcified breast masses. Acta Radiol 1997;38:497-502.

14. Conway WF, Hayes C, Brewer W. Occult breast masses: use of a mammographic localizing grid for US evaluation. Radiology 1991;181:143-146.

15. Richter K, Prihoda H, Heywang-Kobrunner SH, et al. Description and first clinical use of a new system for combined mammography and automated clinical amplitude/velocity reconstructive imaging breast sonography. Invest Radiol 1997;32:19-28.

16. Li B, Thibault J-B, Hall AL. Combining X-ray and ultrasound imaging for enhanced mammography. 2010 Nov;(9) United States Patent and Trademark Office, Patent Number 7,831,015.

17. Kapur A, Carson PL, Eberhard J, et al. Combination of digital mammography with semi-automated 3D breast ultrasound. Technol Cancer Res Treat 2004;3:325-334.

18. Suri JS, Danielson T, Guo Y, et al. Fischer's Fused Full Field Digital Mammography and Ultrasound System (FFDMUS). Stud Health Technol Inform 2005;114:177-200.

19. Schulz-Wendtland R, Wittenberg T, Michel T, et al. Future of mammography-based imaging. Radiologe 2014;54:217-223.

20. Schulz-Wendtland R, Jud SM, Fasching PA, et al. A standard mammography unit - standard 3D ultrasound probe fusion prototype: first results. Geburtshilfe Frauenheilkd 2017;77:679-685.

21. Redondo A, Comas M, Macia F, et al. Inter- and intraradiologist variability in the BI-RADS assessment and breast density categories for screening mammograms. Br J Radiol 2012;85:1465-1470.

22. Poulos A, McLean D, Rickard M, et al. Breast compression in mammography: how much is enough? Australas Radiol 2003;47:121-126.

23. Booi RC, Krucker JF, Goodsitt MM, et al. Evaluating thin compression paddles for mammographically compatible ultrasound. Ultrasound Med Biol 2007;33:472-482. 